\title{
Abschied vom Euro?
}

\section{Philip Arestis Malcolm Sawyer}

\begin{abstract}
Die Eurokrise beherrscht auch in Deutschland die aktuelle wirtschaftspolitische Debatte. Die Krise wird dabei fast ausschließlich als Staatsschuldenkrise gesehen, die wesentlich auf eine exzessive Finanzpolitik zurückgeführt wird. Wir zeigen dagegen, dass die aktuellen Probleme im Euroraum - insbesondere die unhaltbaren Leistungsbilanzungleichgewichte - sich schon seit Beginn des Europrojekts aufgebaut haben und eine Folge des unzureichenden und nicht zweckdienlichen Charakters der europäischen wirtschaftspolitischen Regelungen sind. Kommt es nicht zu tief greifenden Veränderungen in den wirtschaftspolitischen Institutionen, dann ist der Euro nicht mehr zu retten. ${ }^{1}$
\end{abstract}

\section{Einleitung}

Durch Finanzkrise und Rezession traten mehrere Probleme des "Europrojekts" zutage, die eng mit grundsätzlichen Themen der gemeinsamen Währung zusammenhängen. Die Konvergenzkriterien des Maastricht-Vertrages basieren auf rein nominalen anstelle von realen Variablen und lassen Themen wie die Leistungsbilanzsalden völlig außer Acht. Hinzu kommen die bekannten Schwierigkeiten makroökonomischer Politik im Rahmen des Stabilitäts- und Wachstumspaktes (SWP): seine deflationäre Tendenz und das Problem einer undifferenzierten, einheitlichen Defizitgrenze für alle Länder.

Dieser Beitrag beschäftigt sich mit der makroökonomischen Performanz der Euroländer mit besonderem Blick auf die unterschiedlichen Inflationsraten, die Verschiebungen der Wettbewerbsfähigkeit innerhalb des Euroraums und die aktuellen Leistungsbilanzdefizite und ihre Auswirkungen auf die zukünftige Performanz des Euroraums sowie auf den Euro selbst. Es ergibt sich, dass die aktuellen Leistungsbilanzdefizite und -überschüsse im Zusammenhang mit Arbeitslosigkeit, mangelnder Wettbewerbsfähigkeit und öffentlichen Haushaltsdefiziten stehen. Zugleich wird das Wesen der vorgeschlagenen Reformen für den laufenden Betrieb des Euroraums untersucht. Wir diskutieren die politischen (einschließlich der aus dem Lissabon-Vertrag resultierenden) und ideologischen (auf einer neoliberalen Agenda basierenden) Beschränkungen, denen sich eine ernsthafte Reformpolitik gegenübersieht. Dabei kommen wir zu dem Ergebnis, dass die notwendigen tief greifenden Reformen nicht zum Tragen kommen werden. Wir erörtern die Möglichkeiten einer substanziellen Finanzpolitik auf Ebene der Europäischen Union (EU) sowie andere Aspekte einer politischen Union und stellen fest, dass die tief liegenden Probleme wahrscheinlich nicht gelöst werden und dem Euro deshalb eine düstere Zukunft bevorsteht.

Die aktuelle „große Rezession” stellt viele Länder auf der ganzen Welt vor schwere Probleme. Kaum irgendwo sonst sind diese allerdings größer als in den Ländern des Euroraums. Das rasante Anwachsen der öffentlichen Schuldenstände durch den Rückgang der Wirtschaftsleistung und dadurch verminderte Steuereinnahmen führte zum Bruch der Regeln des Stabilitäts- und Wachstumspakts. Glücklicherweise wurde dieser Bruch zunächst allgemein akzeptiert. Aber schon bald wurde nach konzertierten Aktionen zur Reduzierung der Schuldenstände und nach Haushaltskonsolidierung gerufen. Die Versuche der einzelnen Länder, ihre Defizite zurückzufahren, bergen jedoch die Gefahr kumulativer negativer Auswirkungen auf Beschäftigung und Wachstum, ohne dabei nennenswerten Einfluss auf die staatlichen Budgetdefizite zu haben.

Dass enorme Haushaltsdefizite deutlich oberhalb der vom SWP geforderten $3 \%$-Grenze ebenso unvermeidlich wurden wie die damit verbundene faktische zeitweise Aussetzung dieser Grenze, das zeigte unmittelbar die Schwachstellen des SWP auf. Es gibt aber noch eine Reihe weiterer Probleme, die in den politischen Rahmenbedingungen des Euroraums angelegt sind. Sie beruhen weniger auf Vorschriften für eine bestimmte Politik, als vielmehr auf dem Fehlen solcher Vorschriften. So fehlt es an einer wirksamen

1 Dieser Aufsatz ist die Kurzfassung eines Beitrags zur 7. internationalen Konferenz "Developments in Economic Theory and Policy" in Bilbao vom 1.-2. Juli 2010, organisiert vom Department of Applied Economics V der University of the Basque Country und dem Cambridge Centre for Economic and Public Policy, Department of Land Economy, University of Cambridge.

Philip Arestis, Dr., ist Honorary Senior Departmental Fellow am Cambridge Centre for Economic and Public Policy der Universität Cambridge und Professor an der Universität des Baskenlandes in Bilbao sowie Chief Academic Adviser to the UK Government Economic Service (GES) on Professional Developments in Economics. Arbeitsschwerpunkte: Makroökonomische Theorie und Politik. e-mail: pa267@hermes.cam.ac.uk Malcolm Sawyer, Dr., ist Professor of Economics an der University of Leeds. Arbeitsschwerpunkte: Makroökonomie, Finanz- und Geldpolitik, Politische Ökonomie der Europäischen Währungsunion. e-mail: M.C.Sawyer@/ubs.leeds.ac.uk

Übersetzung aus dem Englischen von Yvonne Silber und Achim Truger 
Inflationspolitik, vor allem im Hinblick auf die unterschiedlichen Inflationsraten in den einzelnen Ländern, ebenso wie an einem Ausgleichsprozess für die ständigen Leistungsbilanzdefizite einiger Länder, denen die Überschüsse anderer Länder gegenüberstehen. Aus Letzterem folgt, dass sich die Defizitländer unvermeidlich im Ausland verschulden müssen. Die Interaktion der deflationären Tendenzen des SWP, die fehlende Berücksichtigung der unterschiedlichen Inflationsraten und das folgerichtige Missverhältnis der Wettbewerbsfähigkeiten der verschiedenen Länder sowie das resultierende Muster von Leistungsbilanzdefiziten und -überschüssen ergeben eine toxische Mischung für die wirtschaftlichen Aussichten der Euroländer.

Um die im Europrojekt von Anfang an eingebauten Fehler zu verstehen, die nun das Gebäude der Europäischen Wirtschafts- und Währungsunion (WWU) untergraben, gilt es zunächst die Konvergenzkriterien und den SWP zu untersuchen (Abschnitt 2). Abschnitt 3 liefert mit einer Kurzanalyse der Konvergenzkriterien des Stabilitätspakts den gesetzlichen Rahmen für den Euro. Abschnitt 4 analysiert die wirtschaftliche Entwicklung der WWUMitglieder seit der Euro-Einführung, bevor Abschnitt 5 zu der Frage zurückkehrt, ob die Zeit des Euro abgelaufen ist. Wir stellen einige politische Überlegungen über die notwendigen massiven Änderungen hin zu einem effektiven Funktionieren des Euro an. Der begründete Zweifel an der Umsetzung solcher Reformen führt uns zu skeptischen Schlussfolgerungen (Abschnitt 6).

\section{Die Konvergenzkriterien}

Der Maastricht-Vertrag formuliert eine Reihe von „Konvergenzkriterien“, die die Natur des Europrojekts und die in diesem Rahmen für wichtig und bedeutend gehaltenen Elemente verdeutlichen. Die Übernahme nationaler "unabhängiger“ Zentralbanken als Vorgänger für die Eingliederung in das Europäische System der Zentralbanken mit der Europäischen Zentralbank an der Spitze verweist auf die zugrunde liegende neoliberale Agenda (Lucarelli 2004; Arestis/Sawyer 2006a). Die Anforderung eines staatlichen Haushalts- defizits unterhalb von $3 \%$ des Bruttoinlandprodukts (BIP) und einer öffentlichen Verschuldung unterhalb von $60 \%$ des BIP zeigen deutlich die einseitige Fixierung auf den Saldo des Staatssektors. Dabei wurde die $60 \%$-Grenze von vielen Ländern bereits beim Eintritt in die WWU nicht eingehalten und das $3 \%$-Limit vielfach nur durch den Einsatz „kreativer Buchführung" ermöglicht (Arestis et al. 2001). Die Vorgaben für Zins- und Inflationsraten eines Landes nahe des Durchschnitts der drei Länder mit der niedrigsten Inflationsrate wurden damit begründet, dass nach der Formierung der WWU ein einheitliches Zinsregime herrschen würde und gemeinsame Inflationswerte für den weiteren Erfolg des Euro erforderlich wären. Einen ähnlich intuitiven Charakter hatte die geforderte Wechselkursstabilität eines Landes gegenüber den anderen Ländern der WWU, da die Wechselkurse hier ohnehin kurz vor ihrer endgültigen Fixierung standen.

Die von den Konvergenzkriterien nicht behandelten Themen zeigen auf, was für wichtig und was für unbedeutend gehalten wurde; diese Nichtbeachtung war der Grund für viele der anschließend folgenden Probleme. Eine Annäherung der Konjunkturzyklen, die für die makroökonomische Politik innerhalb der WWU wichtig gewesen wäre, wurde nicht erwähnt. Die faktische Beschränkung auf die Geldpolitik, die nur eine undifferenzierte Politik sein kann, führt unausweichlich zu einem Standardisierungsproblem. Auch das Thema Arbeitslosigkeit wurde nicht beachtet, obwohl niedrige Arbeitslosenquoten schließlich das Ziel der Geldpolitik und jeder anderen Art von Wirtschaftspolitik sind. Doch die unterschiedlichen Arbeitslosenquoten der Länder beim Eintritt in das Euro-System der fixen Wechselkurse wurden außer Acht gelassen. Fixe Wechselkurse zwingen jedoch Länder mit hohen Arbeitslosenquoten, mit den Mitgliedsländern mit relativ niedrigerer Arbeitslosigkeit gleichzuziehen. Während die Konvergenzkriterien ein Zusammenlaufen der Inflationsraten zum WWU-Beitrittszeitpunkt verlangten, machte man sich über entsprechende Inflationsbedingungen wenig Gedanken, wie z.B. über die Frage, ob das Erreichen des Inflationsziels von anfangs 0 bis $2 \%$ pro $\mathrm{Jahr}^{2}$ verschiedene Arbeitslosenquoten erfordern würde. Erstaunlicherweise wurde auch der Frage wenig Beachtung geschenkt, ob der Wechselkurs, mit dem ein Land dem Euro beitrat, nachhaltig sein würde. Es wurde lediglich in Betracht gezogen, ob die betreffende Währung vor Beitritt bereits Mitglied des Europäischen Währungssystems (EWS) war und ihr Wechselkurs daher in der Nähe des zentralen Werts lag. Ob sich die Leistungsbilanz allerdings im Plus oder im Minus befand und ob die daraus folgende Auslandsvermögensposition auf Dauer tragfähig sein würde, war anscheinend nicht von Interesse. Diese Mischung von unterschiedlichen Inflationsbedingungen und Nichtbeachtung der Nachhaltigkeit der einzelnen Leistungsbilanzsalden zum Beitrittszeitpunkt erweist sich nun als schädlich für die WWU. Die Wettbewerbsfähigkeit einiger Länder hat sich seither verschlechtert, was ihre Leistungsbilanzdefizite und die daraus folgende Notwendigkeit, sich im Ausland zu verschulden, noch vergrößert hat. Unter dem Einfluss der „großen Rezession“ wurden schließlich private Auslandsschulden zur öffentlichen Verschuldung.

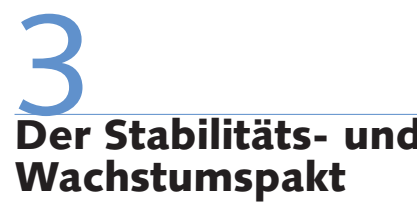

Der Stabilitäts- und Wachstumspakt (SWP) mit seinem finanz- und geldpolitischen Ansatz bildet den Kern des politischen Rahmenwerks der Wirtschafts- und Währungsunion (WWU) und gewährt einen Einblick in das Wesen des Europrojekts.

In der Formulierung: „(1) Die Mitgliedstaaten vermeiden übermäßige öffentliche Defizite“ (Vertrag, Artikel 126 (1)) liegt das Schlüsselkonzept des Stabilitäts- und Wachstumspakts. Der weitere Text lautet: „(2) Die Kommission überwacht die Entwicklung der Haushaltslage und der Höhe des öffentlichen Schuldenstands in den Mitgliedstaaten im Hinblick auf die Feststellung schwerwiegender Fehler. Insbesondere prüft sie die Einhaltung der Haushaltsdisziplin anhand von zwei Kriterien, nämlich daran,

a) ob das Verhältnis des geplanten oder tatsächlichen öffentlichen Defizits zum Bruttoinlandsprodukt einen bestimmten Referenzwert überschreitet, es sei denn, dass entweder das Verhältnis erheblich

\footnotetext{
2 So interpretierte die EZB bis 2003 ihr Ziel der Preis-
} stabilität. 
und laufend zurückgegangen ist und einen Wert in der Nähe des Referenzwerts erreicht hat, oder der Referenzwert nur ausnahmsweise und vorübergehend überschritten wird und das Verhältnis in der Nähe des Referenzwerts bleibt,

b) ob das Verhältnis des öffentlichen Schuldenstands zum Bruttoinlandsprodukt einen bestimmten Referenzwert überschreitet, es sei denn, dass das Verhältnis hinreichend rückläufig ist und sich rasch genug dem Referenzwert nähert." (Artikel $126(2))$.

Protokoll 12 zum Thema exzessive Verschuldung setzt $3 \%$ des Bruttoinlandsprodukts (BIP) als Referenzwert für Haushaltsdefizite und $60 \%$ des BIP für den Staatsschuldenstand fest. Weiterhin wird die Ansicht vertreten: „Das Festhalten an dem Ziel eines soliden, nahezu ausgeglichenen oder einen Überschuss aufweisenden Haushalts wird es allen Mitgliedstaaten ermöglichen, die normalen Konjunkturschwankungen zu bewältigen und dabei das öffentliche Defizit im Rahmen des Referenzwerts von 3 \% des BIP zu halten" (Entschließung des Europäischen Rates über den Stabilitäts- und Wachstumspakt, Amsterdam, 17. Juni 1997, Amtsblatt C 236 vom 2.8.1997, S. 0001-0002).

Es bleibt zunächst festzustellen, dass das Ziel eines über den Zyklus ausgeglichenen Haushalts mit einem realen Budgetüberschuss gleichzusetzen ist, wenn man die Inflation und die dadurch bedingte Verminderung der realen öffentlichen Schuldenlast berücksichtigt: Bei einer Inflationsrate von $2 \%$ und einer Schuldenstandsquote von $60 \%$ ergibt dies einen Wert von 1,2\% des BIP. Eine Schuldenstandsquote von $60 \%$ ist mit einer ständigen Neuverschuldung von ca. $3 \%$ des BIP vereinbar - auf Basis einer nominalen Wachstumsrate von $5 \%$ pro Jahr (sprich $2-3 \%$ reales Wachstum bei $2-3 \%$ Inflation) und der Formel $b=d / g$, wobei $b$ die Staatsverschuldung im Verhältnis zum BIP darstellt, $d$ das Haushaltsdefizit (einschließlich der Zinszahlungen) und $g$ die nominale Wachstumsrate. Die Forderung nach Haushaltsausgleich und gleichzeitigem Schuldenstand von $60 \%$ des BIP ist deshalb von vornherein widersprüchlich.

Nach allgemeiner Erfahrung der EULänder (und der meisten anderen) liegen Staatshaushalte eher im Defizitbereich - anderenfalls hätten viele EU-Länder (auch vor der aktuellen Rezession) nicht Schuldenstände von weit mehr als $60 \%$ des BIP. So lag z.B. das durchschnittliche Haushaltsdefizit in Westdeutschland in den beiden Jahrzehnten von 1970 bis 1991 bei jährlich $2 \%$ des BIP. Der geforderte Ausgleich über den Zyklus fand also nicht statt. Es ist deshalb unerklärlich, warum sich Länder freiwillig finanzpolitische Einschränkungen auferlegten, die schon in der Vergangenheit nicht erfüllt wurden. Solche finanzpolitischen Einschränkungen ergäben nur dann einen ökonomischen Sinn, wenn man zeigen könnte, dass sie erstens mit hoher wirtschaftlicher Aktivität vereinbar und zweitens dieselben Einschränkungen für alle Länder und über die Zeit hinweg gleichermaßen gültig sind. Wer auf der Basis des Say'schen Gesetzes und der Ricardianischen Äquivalenz annimmt, dass ein durchschnittliches Haushaltsdefizit nicht erforderlich sei, der könnte möglicherweise die Anwendung derselben Regel für alle Länder fordern. Wer allerdings kein strenggläubiger Anhänger beider Lehrsätze ist, für den gibt es keine Rechtfertigung, einen ausgeglichenen Haushalt zu verlangen.

Der Stabilitäts- und Wachstumspakt brachte für die Geld- und Finanzpolitik seltsame Neuerungen: auf EU-Ebene ein sehr geringes Ausgabenniveau (knapp oberhalb von $1 \%$ des EU-BIP) und die Anforderung eines streng ausgeglichenen Haushalts (ohne zwischen der Mitgliedschaft in EU oder WWU zu unterscheiden), auf nationaler Ebene das Erfordernis eines ausgeglichenen Gesamthaushalts mit der Erlaubnis, die ,automatischen Stabilisatoren" über den Zyklus wirken zu lassen, obwohl das im Prinzip durch die 3-\%-BIPGrenze eingeschränkt wurde. Warum ein ausgeglichener Staatshaushalt aber mit hoher Beschäftigung und hohem Wachstum vereinbar sein soll, wurde nicht erklärt.

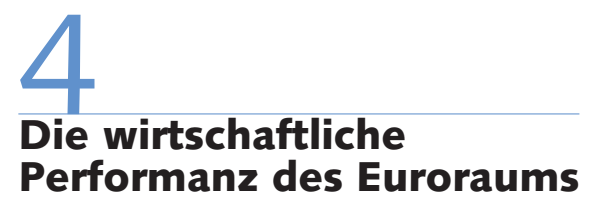

Im Folgenden wird die wirtschaftliche Performanz der Länder des Euroraums im Hinblick auf Wachstum, Arbeitslosigkeit, Inflation und Leistungsbilanz sowie die Frage, in welchem Maße die Bestimmungen des Stabilitäts- und Wachstumspakts eingehalten wurden, untersucht. Besonders prüfen wir die unterschiedlichen Inflationsraten, die Änderungen bei der Wettbewerbsfähigkeit und die Leistungsbilanzdefizite mit ihren Auswirkungen auf die Zukunft der Wirtschaftsleistung in Euroland und für den Euro selbst. Denn die aktuellen Niveaus von Leistungsbilanzdefiziten und -überschüssen hängen eng mit den Themen Arbeitslosigkeit, mangelnde Wettbewerbsfähigkeit und Haushaltsdefizite zusammen.

Die Tabellen 1 und 2 zeigen Indikatoren für die wirtschaftliche Performanz des Euroraums im ersten Jahrzehnt nach Einführung des Euro als „,echte“ Währung (nach drei Jahren seines Bestehens als „virtuelle"Währung). Ein Blick auf die Daten in Tabelle 1 (sowie auf weitere, umfassendere Daten) zeigt uns vor allem Folgendes:

(1) Selbst in den Jahren von 2002 bis 2007, im globalen Wirtschaftsboom, lag das durchschnittliche Haushaltsdefizit bei jährlich 2,2 \% des BIP (2008 und 2009 hinzugerechnet, steigt der Wert auf 2,7\%). Vier Länder hatten einen durchschnittlichen Haushaltsüberschuss (Finnland, Luxemburg, Irland und Spanien); erweitert man den Berechnungszeitraum bis 2009, wiesen die beiden letztgenannten Länder jedoch ein durchschnittliches Defizit auf.

(2) Zwischen den einzelnen WWU-Mitgliedern bestanden große und andauernde Leistungsbilanzunterschiede.

(3) Die Inflationsrate lag oberhalb des 0 bis 2-\%-Bereichs, der als Preisstabilität definiert ist, wenn auch nur knapp. Die Inflationsrate lag in jedem Jahr des neuen Jahrzehnts zwischen 2 und 2,5\%, bis sie 2008 auf 3,3\% stieg.

(4) Zwischen den Ländern gab es große Unterschiede bei den Veränderungen der Lohnstückkosten und damit in der Wettbewerbsfähigkeit.

Hinzu kommt, dass die Ziele für die öffentlichen Schuldenstandsquoten allgemein verfehlt wurden. Selbst vor Ausbruch der Rezession hatten nur die oben genannten vier Länder und die Niederlande einen öffentlichen Schuldenstand unterhalb von $60 \%$ des BIP. Diese Ergebnisse trugen wenig zur Glaubwürdigkeit des Euroraums bei, die ja das Ziel des SWP gewesen war.

Der Stabilitäts- und Wachstumspakt wurde eingeführt, um die Vertrauenswürdigkeit des Euro zu fördern. In den Jahren 2000 bis 2007 lag die Wachstumsrate 
im Euroraum etwas niedriger als in den 1990er Jahren (siehe Tabelle 2), obwohl die Weltwirtschaft insgesamt schneller wuchs. Wenn man die Jahre 2008 und 2009 einbezieht, fällt der Vergleich zwischen den 1990er und den 2000er Jahren für das Europrojekt noch ungünstiger aus. Die Arbeitslosigkeit sank Mitte der 2000er Jahre, dieser Erfolg wurde jedoch durch die Rezession wieder zunichtegemacht. Die Zahlen veranschaulichen die Unterschiede in den Leistungsbilanzen zwischen den einzelnen Ländern. Die südlichen Euroländer weisen große Defizite, die nördlichen dagegen Überschüsse auf.

Die Ergebnisse der Geldpolitik im letzten Jahrzehnt verdeutlichen, dass die Europäische Zentralbank (EZB) nicht in der Lage war, das Inflationsziel von 0 bis $2 \%$ pro Jahr im Euroraum zu erreichen. Dieses Inflationsziel wurde dann im Mai 2003 in „unterhalb, aber nahe $2 \%$ “ geändert. Das Ausmaß der Zielverfehlung war zwar klein, aber beständig. 2008 stieg die Inflationsrate jedoch auf über $3 \%$. Dies konnte als durch die globalen Öl- und Lebensmittelpreise hervorgerufene, kostengetriebene Inflation interpretiert werden. Die Geldpolitik kann allerdings bestenfalls eine Nachfrageinflation bekämpfen, während sie bei kostengetriebener Inflation machtlos ist.

Die unterschiedlichen Inflationsraten der einzelnen Mitgliedsländer veranschaulichen das Problem der einheitlichen Geldpolitik. Wenn die der Geldpolitik und insbesondere dem Inflation-Targeting zugrunde liegenden Argumente korrekt sind ${ }^{3}$, haben die geldpolitischen Aktivitäten vermutlich das Problem noch verschlimmert. Einem Inflation-Targeting durch zinssetzende Geldpolitik liegt die Annahme zugrunde, dass einer drohenden hohen Inflation mit einem Anstieg des nominalen Zinssatzes begegnet werden kann, was wiederum zu einem Anstieg des Realzinssatzes führt. Dadurch soll die Nachfrage und damit die Inflation gedämpft werden. Ein von der EZB festgelegter, für alle Euroraumländer gleichermaßen geltender Zinssatz führt jedoch in einem Land mit relativ hoher Inflationsrate zu einem relativ niedrigeren Realzins. Das bedeutet aber gemäß dem geldpolitischen Transmissionsmechanismus eine höhere statt niedrigere Inflationsrate.

Wie weiter oben erläutert, war die Inflationspolitik bestenfalls ein bescheidener Erfolg. Dazu kommt, dass es eher dem Glücksfall eines international niedrigen In-

Tabelle 1: Wirtschaftliche Performanzindikatoren zur Stabilität des Euroraums - in \% -

\begin{tabular}{lcccc}
\hline & $\begin{array}{c}\text { Haushaltsüberschuss/ } \\
\text { BIP: Durchschnitt } \\
\mathbf{2 0 0 2 - 2 0 0 7}\end{array}$ & $\begin{array}{c}\text { Leistungsbilanz- } \\
\text { saldo/BIP } \\
\mathbf{2 0 0 2 - 2 0 0 9}\end{array}$ & $\begin{array}{c}\text { Jährliche } \\
\text { Inflationsrate } \\
\mathbf{2 0 0 2 - 2 0 0 8}\end{array}$ & $\begin{array}{c}\text { Entwicklung der } \\
\text { Lohnstückkosten in Dollar } \\
\mathbf{2 0 0 1 - 2 0 0 8}\end{array}$ \\
\hline Österreich & $-1,9$ & 2,5 & 2,1 & 3,5 \\
Belgien & $-0,6$ & 2,0 & 2,3 & 12,0 \\
Finnland & 3,4 & 4,5 & 1,8 & 4,3 \\
Frankreich & $-3,2$ & $-0,4$ & 2,2 & 7,7 \\
Deutschland & $-2,7$ & 4,8 & 1,9 & 5,4 \\
Griechenland & $-5,1$ & $-9,7$ & 3,7 & 17,0 \\
Irland & 1,1 & $-2,8$ & 2,9 & 34,6 \\
Italien & $-3,2$ & $-2,0$ & 2,6 & 9,7 \\
Luxemburg & 1,1 & 8,6 & 3,0 & 9,5 \\
Niederlande & $-1,1$ & 6,5 & 2,2 & 11,4 \\
Portugal & $-3,7$ & $-9,1$ & 2,7 & 7,4 \\
Spanien & 0,6 & $-6,7$ & 3,3 & 16,5 \\
Durchschnitt & $-2,2$ & 0,3 & 2,4 & \\
Euroraum & & & &
\end{tabular}

Quelle: OECD Economic Outlook Datenbank, Berechnungen der Autoren.

WSI MITTEILUNGEN

Tabelle 2: Wachstum, Arbeitslosigkeit und Leistungsbilanzen im

Euroraum - in \% -

\begin{tabular}{|c|c|c|c|c|c|}
\hline & $\begin{array}{c}\text { Wachstum } \varnothing \\
1991-2000\end{array}$ & $\begin{array}{l}\text { Wachstum } \varnothing \\
2000-2007\end{array}$ & $\begin{array}{l}\text { Arbeitslosen- } \\
\text { quote } 2000\end{array}$ & $\begin{array}{l}\text { Arbeitslosen- } \\
\text { quote } 2009\end{array}$ & $\begin{array}{l}\text { Leistungsbilanz- } \\
\text { saldo (\% des BIP) }\end{array}$ \\
\hline Österreich & 2,3 & 1,9 & 4,8 & 5,8 & 1,9 \\
\hline Belgien & 2,3 & 1,6 & 6,9 & 7,9 & $-0,8$ \\
\hline Finnland & 2,9 & 2,1 & 9,8 & 8,3 & 0,8 \\
\hline Frankreich & 2,1 & 1,5 & 8,6 & 9,1 & $-2,0$ \\
\hline Deutschland & 1,7 & 0,7 & 7,4 & 7,6 & 4,0 \\
\hline Griechenland & 2,3 & 4,3 & 11,4 & 9,3 & $-11,1$ \\
\hline Irland & 7,7 & 3,9 & 4,3 & 11,9 & $-2,8$ \\
\hline Italien & 1,6 & 0,3 & 10,2 & 7,6 & $-2,7$ \\
\hline Luxemburg & 4,6 & 3,7 & 2,6 & 5,9 & 1,9 \\
\hline Niederlande & 3,2 & 1,6 & 2,8 & 3,7 & 6,2 \\
\hline Portugal & 2,7 & 0,7 & 4,0 & 9,2 & $-9,7$ \\
\hline Spanien & 2,8 & 3,0 & 10,8 & 18,1 & $-5,3$ \\
\hline EU-12 & 2,1 & 1,3 & 8,2 & 9,4 & $-0,5$ \\
\hline
\end{tabular}

flationsumfelds in der meisten Zeit der zurückliegenden Dekade als der Wirksamkeit dieses Politikinstruments zu verdanken ist, dass die Inflationsrate in der Nähe, aber doch oberhalb von $2 \%$ gehalten werden konnte. Nach unserer Auffassung wird die Inflation in der WWU und anderswo nur in begrenztem Ausmaß von der einheimischen Politik beeinflusst. Es gab zwar die WWU-Inflationspolitik der EZB, aber auch eine Inflationspolitik auf nationaler Ebene. Einzelne Länder betreiben auf nationaler Ebene eine mehr oder weniger unterschiedliche Lohn- und Preispolitik. Wie oben beschrieben, blieben die Unterschiede der nationalen Inflationsraten entweder durch nationale Politik oder durch Unterschiede im Preis- und Lohnniveau bestehen.

Bleiben diese Inflationsunterschiede weiterhin bestehen, wird der Euro weiter ausgehöhlt. Es ist offensichtlich, dass es derzeit kein politisches Instrument auf EU-Ebene gibt, das diese Problemlage in den Griff bekäme. Man könnte behaupten, dass der Integrationsdruck zu ähnlichen Inflationsraten in den einzelnen Ländern führt. Selbst wenn dies der Fall wäre, könnten ähnliche Inflationsraten immer noch an unterschiedliche Arbeitslosenquoten gekoppelt sein. Paradoxerweise könnte das Zusammenlaufen der Inflationsraten durch die nationale Finanzpolitik gesteuert werden (allerdings empfehlen wir diesen Weg nicht). Unentbehrlich hierfür ist jedenfalls die Entwicklung eines gemeinsamen Verständnisses der WWU-Länder zu diesem Thema.

3 Unsere Zweifel an der Politik des Inflation-Targeting beschreiben wir in Arestis/Sawyer (2008). 
Tabelle 1 gibt einen Hinweis auf das Ausmaß der Entwicklung der relativen Preise und der relativen Lohnstückkosten seit Fixierung der Wechselkurse der ursprünglichen nationalen Währungen der WWU-Mitglieder untereinander. Diese Veränderungen können als Anpassung der realen Wechselkurse zwischen den WWU-Mitgliedern in Kombination mit unangemessen festgelegten nominalen Wechselkursen (für die meisten Länder geschah dies 1998) und auf „Schocks“ interpretiert werden. Diese Sichtweise lässt allerdings die zurzeit der Euro-Einführung herrschenden Leistungsbilanzdefizite und deren Tendenz zur Verfestigung und Ausweitung außer Acht.

Ein Land in einem fixen Wechselkurssystem (das Wesen einer Währungsunion für die teilnehmenden Länder) kann angesichts wachsender Inflation und aufgelaufener Leistungsbilanzdefizite in Deflation verfallen (durch Reduzierung der Importe und eventuell inländische Kostensenkungen), oder es kann seine Währung abwerten. Letzteres ist allerdings durch die Mitgliedschaft in der WWU ausgeschlossen. Ist Deflation also der einzig mögliche Weg? Ein Leistungsbilanzdefizit kann mit einem Haushaltsdefizit auf folgende Weise interagieren: Leistungsbilanzdefizit und Haushaltsdefizit werden bei einer gegebenen Nettosparquote gegenübergestellt. Bleiben andere Faktoren konstant (die Sparquote), wird ein höheres Leistungsbilanzdefizit mit einem höheren Haushaltsdefizit einhergehen (obwohl dies keine kausale Verbindung impliziert).

Die „unabhängige” Europäische Zentralbank beschränkt sich mit ihrer Inflationspolitik auf ,ein einziges Instrument und ein einziges Ziel“". Die Fokussierung auf die Inflation als "einziges Ziel“ wurde vielfach kritisiert, weil sie einen hohen Beschäftigungsstand und ein hohes Produktionsniveau als Ziele makroökonomischer Politik ausblendet (und viele andere Variablen gleichermaßen - der Schwerpunkt liegt hier allerdings auf der Beschäftigung bzw. Arbeitslosigkeit). Allerdings kann dieser Kritik im Rahmen des Phillips-Kurven-Ansatzes scheinbar leicht begegnet werden. Diesem zufolge muss das angestrebte Produktionsniveau mit konstanter Inflation vereinbar sein. Wird die wirtschaftliche Aktivität nach Beschäftigungskriterien beurteilt, wäre das Ziel die inflationsstabile Arbeitslosenquote (NAIRU), und bei Betrachtung der Produktionsleistung müsste die Produktionslücke gleich null sein, d.h. die aktuelle Produktionsleistung müsste dem Trendwert oder Produktionspotenzial entsprechen. Jedes andere Niveau der Wirtschaftsleistung würde zu stetig steigender oder fallender Inflation führen. Allerdings werden inflationsstabile Arbeitslosenquote und Nullwert-Produktionslücke von angebotsseitigen Gleichgewichtsansätzen unterstellt, und, was viel wichtiger ist, sowohl kurz- als auch langfristig nicht von der Gesamtnachfrage und der Wirtschaftsleistung beeinflusst. An anderer Stelle haben wir argumentiert, dass die wirtschaftliche Aktivität (durch Kapazitätsauslastung und Ertragskraft) das Investitionsniveau beeinflusst, was wiederum eine Erhöhung des Kapitalstocks und der Produktionskapazitäten zur Folge und damit Einfluss auf die inflationsstabile Arbeitslosenquote hat (so z.B. Sawyer 2002; Arestis/Sawyer 2005).

\section{Leb wohl, Euro?}

Die oben ausgeführten Argumente resultieren unmittelbar aus der schlechten Wirtschaftsentwicklung im Euroraum bzw. aus den Folgen der Finanzkrise, die die inneren Probleme des Europrojekts zum Vorschein gebracht haben. Der Schluss liegt also nahe, dass für den Euro mit großer Wahrscheinlichkeit die Zeit gekommen ist, abgelöst zu werden oder unterzugehen. Es stellt sich nun die bedeutende Frage, inwieweit er gerettet werden kann.

\subsection{IST DER EURO NOCH ZU RETTEN?}

Der Euro kann als auf zwei Säulen stehendes Projekt betrachtet werden. Die eine Säule ist ein im Wesentlichen neoliberal geprägtes politisches Rahmenwerk. Die andere Säule betrachtet die Einheitswährung als Endstadium wirtschaftlicher Integration, die das vermutlich letzte Hindernis zum Freihandel beseitigt (unterschiedliche Währungen und die damit verbundenen Kosten), nachdem die außertariflichen Handelshemmnisse bereits durch die Bestimmungen der Einheitlichen Europäischen Akte abgeschafft worden waren.

Die Elemente der ersten Säule wurden in den verschiedenen Verträgen der Europäischen Union verankert und im Vertrag von Lissabon („Vertrag über die
Arbeitsweise der Europäischen Union“) endgültig festgeschrieben. Änderungen des Lissabon-Vertrages erfordern die einstimmige Zustimmung aller 27 Mitgliedsländer. Da die Stützung des Euro politische Maßnahmen erfordert, die als Schritte in Richtung einer politischen Union zu werten sind, ist die Wahrscheinlichkeit einer Realisierung dieser Maßnahmen damit nahezu ausgeschlossen. Hier zeigt sich nicht nur der Unverstand des politischen Rahmenwerks, sondern auch der Irrsinn, wirtschaftspolitische Regeln in eine Verfassung einzubinden, die praktisch unveränderlich ist. Weiterhin ist festzuhalten, dass die herrschenden makroökonomischen Institutionen der WWU, vor allem die EZB und die Generaldirektion für Wirtschaft und Finanzen, vollständig der neoliberalen Agenda zu folgen scheinen.

Was die zweite Säule betrifft, so wurde von einigen Befürwortern des Euro zwar zugestanden, dass die wirtschaftliche Integration bis dato in vielerlei Hinsicht nicht weit genug fortgeschritten sei, um eine Einheitswährung zu unterstützen. Sie gingen jedoch davon aus, dass die Einführung der Einheitswährung die Integration zu einem Stadium führen würde, das die Einheitswährung schließlich tragen würde. Die in der Literatur zum optimalen Währungsraum beschriebenen Bedingungen können als Wesen der integrationsfördernden Entwicklungen der relativen Preise bei Faktormobilität betrachtet werden. ${ }^{4}$

Die politischen Grenzen (einschließlich der aus dem Lissabon-Vertrag resultierenden) und die ideologischen Beschränkungen (der neoliberalen Agenda) für wichtige Reformen werden weiter unten diskutiert. Die allgemeine Schlussfolgerung lautet, dass es zu den erforderlichen Reformen nicht kommen wird.

\subsection{REFORMEN FÜR DIE WIRT- SCHAFTS- UND WÄHRUNGSUNION}

Einige der notwendigen Änderungen im politischen Rahmenwerk und den WWUStrukturen sollen hier aufgeführt werden. ${ }^{5}$

4 Die Literatur zum optimalen Währungsraum beginnt mit Mundell (1961), McKinnon (1963) und Kenen (1969). Im Überblick siehe z.B. Baldwin/ Wyplosz (2009, Kapitel 11).

5 Dieses Unterkapitel ist sehr kurz gehalten, da andere Beiträge in diesem Schwerpunktheft das Thema "Reformperspektiven“ ausführlich behandeln. 


\section{FINANZPOLITIK}

In der WWU-Finanzpolitik sind zwei grundlegende Umgestaltungen unumgänglich. Dazu gehört zum einen die Einführung einer Finanzpolitik auf EUEbene, die eine erhebliche Ausweitung des EU-Haushalts gestattet und damit die EU in die Lage versetzt, Haushaltsdefizite (oder -überschüsse) zu generieren und so die Wirtschaft in der EU zu stützen. Hierbei liegt der Schwerpunkt auf dem Euroraum, sodass eine EU-Finanzpolitik auf die WWU-Mitglieder beschränkt werden kann. Die Bandbreite einer solchen Politik wurde verschiedentlich festgelegt auf 7, $5 \%$ des BIP (EU-Kommission 1977), $5 \%$ (Huffschmid 2005, Kapitel 16), oder 2 bis $3 \%$ des BIP (Currie 1997; Goodhart/ Smith 1993). Diese EU-Finanzpolitik wird im Allgemeinen nur EU-weiten „Schocks“ begegnen können. Die aktuelle Krise könnte als so ein EU-weiter Schock gewertet werden (auch wenn ein solcher nur einmal in mehreren Jahrzehnten vorkommt). Maßnahmen in der genannten Dimension könnten auf einen solchen Schock allerdings nicht wirksam reagieren, es sei denn in Verbindung mit großen Defiziten auf nationaler Ebene.

Das zweite Element einer finanzpolitischen Umgestaltung soll jedem Mitgliedsland gestatten, seine eigenen finanzpolitischen Impulse nach eigenem Urteil zu seinem Vorteil zu bestimmen. Die Befürchtungen eines „Überlaufeffekts“ (spill-over) der Defizite eines Landes auf die Kreditwürdigkeit und die Zinssätze anderer Länder haben sich als übertrieben herausgestellt. Ohne EU-weite Finanzpolitik, die auf hohe Wirtschaftskraft abzielt, sollte jedes Land frei sein, dieses Ziel anzustreben (so es dies wünscht).

\section{DIE EUROPÄISCHE ZENTRALBANK}

Fundamentale Änderungen sind auch bei der Europäischen Zentralbank nötig. Die EZB (und das ESZB, das Europäische System der Zentralbanken) wurden als „unabhängige“ Zentralbank gegründet. Die „Unabhängigkeit“ einer Zentralbank beruht auf der Vorstellung, dass Politikern bei den Schlüsselelementen makroökonomischer Politik nicht zu trauen sei. Gewählte Politiker würden eher expansive Politik betreiben, ohne sich über die inflationären Folgen Sorgen zu machen. Die „Unabhängigkeit“ der EZB scheint eine Zusammenarbeit und Koordination zwischen den verschiedenen makroökonomisch verantwortlichen Institutionen geradezu auszuschließen. Eine Welt mit vielfältigen Zielen (hohes Produktionsund Beschäftigungsniveau, finanzielle Stabilität, Inflation etc.) braucht vielfältige Instrumente, die in verschiedenen Institutionen beheimatet sind. Diese müssen offensichtlich zusammenarbeiten können. Gegenwärtig spielt die Finanzpolitik im Verhältnis zur vorherrschenden Geldpolitik nur eine untergeordnete Rolle; sie ist machtlos durch das Fehlen einer gemeinsamen WWU-Finanzpolitik und wegen der einseitigen Fokussierung des Stabilitätspakts auf die nationalen Haushaltsdefizite. Die dem zugrunde liegenden Vorstellungen basieren teilweise auf den Grundgedanken der Phillips-Kurve zum kurzfristigen trade-off zwischen Arbeitslosigkeit und Inflation bei gleichzeitigem Fehlen eines langfristigen trade-offs (Sawyer 2010).

Die Finanzkrise hat zumindest eines deutlich gemacht: Finanzielle Stabilität muss Kernziel der makroökonomischen Politik und der Geldpolitik sein. Finanzielle Stabilität sollte zum vorrangigen Ziel erklärt und die Unabhängigkeit der EZB abgeschafft werden. Das Erreichen von finanzieller Stabilität erfordert die Entwicklung einer Reihe von politischen Instrumenten. Die wichtigsten Reformen für die EZB lauten:

(1) Die Aufgaben der EZB sind neu zu formulieren. Hohe und nachhaltige Werte für Beschäftigung, Wirtschaftswachstum und finanzielle Stabilität sind als Ziele aufzunehmen.

(2) Die EZB muss dem Europäischen Parlament gegenüber Rechenschaft ablegen, ihre Statuten müssen dahingehend geändert werden, dass sie in die Koordination zwischen Finanz- und Geldpolitik einbezogen wird und Weisungen anderer europäischer Institutionen wie des Rats für Wirtschaft und Finanzen (ECOFIN) entgegennehmen kann.

(3) Gegenüber nationalen Regierungen innerhalb der WWU operiert die EZB wie eine nationale Zentralbank. Sie soll vor allem in die Lage versetzt werden, die Defizite nationaler Regierungen zu monetisieren.

\section{POLITISCHE UNION}

Wir haben bereits an anderer Stelle ausgeführt (Arestis et al. 2003; Arestis/Sawyer 2006b), dass Währungsunionen, die nicht auch politische Unionen waren, im Allgemeinen keine lange Überlebensdauer hatten. Nur in sehr kleinen Ländern (wie z.B. die ostkaribische Währungsunion, die eine Bevölkerung von einer halben Million Menschen abdeckt) sind die Überlebenschancen günstiger. Aus Sicht der Chartalistischen Geldtheorie hat eine Währungsunion zumindest auch ein Element einer politischen Union, denn es sind die Regierungen, die festlegen, was als gesetzliches Zahlungsmittel gilt und somit zur Zahlung von Steuern akzeptiert wird.

Die Notwendigkeit einer wirksamen gemeinsamen WWU-Finanzpolitik (die wegen der unterschiedlichen Zusammensetzung von EU und WWU allerdings einige Probleme bekäme) wurde oben bereits veranschaulicht. Die Einführung einer solchen Politik würde bedeuten, dass die Höhe von Steuereinnahmen und öffentlichen Ausgaben der WWU-Finanzpolitik unterliegt und der Ausgleich (Haushaltsdefizit bzw. -überschuss) aufWWU-Ebene erfolgt.

Das in einigen WWU-Ländern zu beobachtende Niveau der Leistungsbilanzdefizite ist nicht nachhaltig. Aber die Länder sind in einem System fester nominaler Wechselkurse eingeschlossen und haben daher Verluste in ihrer Wettbewerbsfähigkeit und entsprechend gestiegene reale Wechselkurse hinnehmen müssen. Zum Ausgleich dieser unterschiedlichen Wechselkurse müssen neue Mechanismen gefunden werden, wie z.B. ein koordinierter Ausgleichsmechanismus für die vorherrschenden Wechselkurse zwischen den einzelnen WWU-Ländern und zur Erzielung ähnlicher Inflationsraten.

Wie oben bereits dargelegt, sollte die EZB gegenüber den nationalen Regierungen der Mitgliedsländer und ihren finanziellen Verpflichtungen ähnlich agieren können wie eine nationale Zentralbank gegenüber ihrer nationalen Regierung. Diese politischen Initiativen kommen einer politischen Union bereits sehr nahe. 
Möglichkeit, finanzpolitische Maßnahmen zur Stabilisierung der Wirtschaft zu entwickeln und anzuerkennen, dass Haushaltsdefizite im Allgemeinen hierzu notwendig sind. Drittens gibt es keinen Mechanismus zur Lösung der aktuellen Leistungsbilanzdefizite bzw. -überschüsse, die in ihrer jetzigen Form unhaltbar sind. Ohne die Möglichkeit von Wechselkursanpassungen bleibt den Ländern mit Leistungsbilanzdefiziten nur der Weg in die Deflation.

Der WWU fehlt jeglicher Mechanismus zur Lösung der Defizitprobleme für die einzelnen Länder. Viele WWU-Länder stehen daher vor der drastischen Wahl, in-

nerhalb des Euroverbunds zu verbleiben und einer trüben Zukunft mit Deflation und hoher Arbeitslosigkeit entgegenzusehen oder den Euroraum zu verlassen. Die wirtschaftlichen Probleme haben sich seit der Gründung des Euroraums aufgebaut und sind mit der "großen Rezession“ akut geworden. Die Fehler liegen in der neoliberalen Ausrichtung des Europrojekts, die im Lissabon-Vertrag quasi in Stein gemeißelt ist. Für grundlegende Änderungen des Konzepts bestehen wegen der erforderlichen Einstimmigkeit wenig Chancen. Ohne tief greifende und wesentliche Veränderungen aber blicken viele, wenn nicht alle Länder des Euroraums, in eine

\section{trostlose wirtschaftliche Zukunft.} makroökonomischen Institutionen zu-
sammenzuarbeiten. Zweitens gibt es keine
Das politische Rahmenwerk (Stabilitätseingebettet ist, ist völlig unzureichend und nicht zweckdienlich. Drei Aspekte stechen der EZB hindert sie daran, men und zum Erreichen ander wie hoher Wirtschaftskraft, mit anderen

\section{LITERATUR}

Arestis, P./Brown, A./Sawyer, M. (2001): The Euro: Evolution and Prospects, Cheltenham

Arestis, P./de Paula, L. F./Ferrari, F./Sawyer, M. (2003): The Euro and the EMU: Lessons for Mercosur, in: Arestis, P./Fernando de Paula, L. (Hrsg.): Monetary Union in South America: Lessons from EMU, Aldershot, S. 14-38

Arestis, P./Sawyer, M. (2005): Aggregate Demand, Conflict and Capacity in the Inflationary Process, in: Cambridge Journal of Economics 29 (6), S. 959-974

Arestis, P./Sawyer, M. (2006a): Macroeconomic Policy and the European Constitution, in: Arestis, P./Sawyer, M. (Hrsg.): Alternative Perspectives on Economic Policies in the European Union, Basingstoke, S. 1-36

Arestis, P./Sawyer, M. (2006b): Reflections on the Experience of the Euro: Lessons for the Americas, in: Vernengo, M. (Hrsg.): Monetary Integration and Dollarization: No Panacea, Aldershot, S. 13-27

Arestis, P./Sawyer, M. (2008): A Critical Reconsideration of the Foundations of Monetary Policy in the New Consensus Macroeconomics Framework, in: Cambridge Journal of Economics 32 (5), S. 761-779 Baldwin, R./Wyplosz, C. (2009): The Economics of European Integration, London

Commission of the European Communities (CEC) (1977): Report of the Study Group on the Role of Public Finances in European Integration, Economic and Financial Series A13, Brüssel
Currie, D. (1997): The Pros and Cons of EMU, London

Goodhart, C. A. E./Smith, S. (1993): Stabilization, in: The Economics of Community Public Finance, European Economy: Reports and Studies (5), S. 417-455

Huffschmid, J. (Hrsg.) (2005): Economic Policy for a Social Europe. A Critique of Neo-Liberalism and Proposals for Alternatives, Basingstoke Kenen, P. (1969): The Theory of Optimum Currency Areas, in: Mundell, R./Swoboda, A. (Hrsg.): Monetary Problems of the International Economy, Chicago, S. 41-60

Lucarelli, B. (2004): European Monetary Union: A Neo-Liberal Trojan Horse?, in: Contributions to Political Economy 23 (1), S. 81-91

McKinnon, R. (1963): Optimal Currency Areas, in: American Economic Review 53 (4), S. 717-725

Mundell, R. (1961): A Theory of Optimum Currency Areas, in: American Economic Review (4) 51, S. 657-665

Sawyer, M. (2002): The NAIRU, Aggregate Demand and Investment, in: Metroeconomica 53 (1), S. 66-94

Sawyer, M. (2010): „Phillips"curve, independence of central banks and inflation targetting, in: Fontana, G./McCombie, J./Sawyer, M. (Hrsg.): Macroeconomics, Finance and Money, Essays in Honour of Philip Arestis, Basingstoke, S. 126-138 